AN. MED. INTERNA (Madrid) Vol. 22, N. $^{\circ} 10$, pp. $465-468,2005$

\title{
Enfermedad tromboembólica venosa en un área rural del sur de Extremadura (España)
}

\author{
J. M. CALVO ROMERO, J. C. ARÉVALO LORIDO, J. ROMERO REQUENA, \\ J. L. PÉREZ ALONSO, C. ORTIZ DESCANE, C. GUTIÉRREZ MONTAÑO, \\ J. CARRETERO GÓMEZ
}

Servicio de Medicina Interna. Hospital de Zafra. Zafra, Badajoz

VENOUS THROMBOEMBOLIC DISEASE IN A RURAL AREA OF SOUTHERN EXTREMADURA (SPAIN)

\section{RESUMEN}

Objetivo: Estudiar las características de la enfermedad tromboembólica venosa (ETV) en un área rural del sur de Extremadura (España).

Material y métodos: Estudio observacional prospectivo de todos los pacientes diagnosticados mediante métodos objetivos de ETV en el único hospital de nuestro área entre noviembre de 2002 y noviembre de 2004.

Resultados: Se diagnosticaron 76 casos de ETV: 35 (46,1\%) con tromboembolismo pulmonar (TEP) y $41(53,9 \%)$ con trombosis venosa profunda (TVP) aislada. La edad media fue 72,4 años (rango 35-94 años), $51(67,1 \%)$ tuvieron más de 70 años, y $43(56,6 \%)$ fueron mujeres. La incidencia anual de ETV fue de 0,63 por 1.000 habitantes $(0,74$ por 1.000 habitantes en mujeres y 0,54 por 1.000 habitantes en hombres). Treinta y siete pacientes $(48,7 \%)$ presentaron ETV "idiopática". Tras un seguimiento medio de 5,5 meses con tratamiento anticoagulante, 13 pacientes $(17,1 \%)$ habían fallecido. La existencia de comorbilidad y ETV no "idiopática" fueron significativamente más frecuentes en los pacientes fallecidos. Tres pacientes $(3,9 \%)$ tuvieron una probable recidiva, y todos fallecieron. Hubo 4 casos $(5,3 \%)$ de hemorragia grave no fatal. Nueve pacientes con TVP aislada no precisaron ingreso hospitalario, y no hubo ningún evento en el seguimiento.

Conclusiones: La incidencia de ETV puede ser menor en nuestro área que en otras zonas geográficas. La ETV afecta más frecuentemente a ancianos y a mujeres, y casi la mitad de los casos son "idiopáticos". La mortalidad es elevada, y se relaciona con la existencia de comorbilidad y ETV no "idiopática". Las recidivas y las hemorragias graves no son excepcionales durante el tratamiento anticoagulante. El tratamiento ambulatorio de la TVP aislada puede ser una opción en pacientes seleccionados.

PALABRAS CLAVE: Enfermedad tromboembólica venosa. Tromboembolismo venoso. Epidemiología. España.

\section{ABSTRACT}

Objective: To study the characteristics of the venous thromboembolic disease (VTD) in a rural area of Southern Extremadura (Spain).

Material and methods: Prospective observational study of all patients diagnosed by objective methods of VTD in the only hospital in our area from November 2002 to November 2004.

Results: Seventy-six patients were diagnosed of VTD: 35 (46.1\%) with pulmonary thromboembolism and 41 (53.9\%) with isolated deep vein thrombosis $(D V T)$. The median age was 72.4 years (range 35-94 years), $51(67.1 \%)$ were older than 70 years, and $43(56.6 \%)$ were women. The annual incidence of VTD was 0.63 per 1,000 persons $(0.74$ per 1,000 persons in women and 0.54 per 1,000 persons in men). Thirtyseven patients (48.7\%) had "idiopathic" VTD. After a median follow-up of 5.5 months with anticoagulant therapy, 13 patients $(17.1 \%)$ had dead. Comorbidity and non "idiopathic" VTD were significantly more frequent in the dead patients. Three patients $(3.9 \%)$ presented probable recurrence, and all of them died. There were 4 cases (5.3\%) of non-fatal severe hemorrhage. Nine patients with isolated DVT did not require hospitalization, and there was no event in the follow-up.

Conclusions: The incidence of VTD may be lower in our area than in other geographical areas. VTD affects more frequently to elderly and women, and almost half of cases are "idiopathic". The mortality is high, and it is related to the existence of comorbidity and non "idiopathic" $V T D$. The recurrences and the severe hemorrhages are not exceptional during the anticoagulant therapy. The ambulatory treatment of isolated DVT may be an option in selected patients.

KEY WORDS: Venous thromboembolic disease. Venous thromboembolism. Epidemiology. Spain.

Calvo Romero JM, Arévalo Lorido JC, Romero Requena J, Pérez, Alonso JL, Ortiz Descane C, Gutiérrez, Montaño C, Carretero Gómez J. Enfermedad tromboembólica venosa en un área rural del sur de Extremadura (España). An Med Interna (Madrid) 2005; 22: 465-468.

\section{INTRODUCCIÓN}

La enfermedad tromboembólica venosa (ETV), que incluye la trombosis venosa profunda (TVP) y el tromboembolismo pulmonar (TEP), es relativamente frecuente y es causa de importante morbilidad y mortalidad $(1,2)$. En nuestro conocimiento, no existen estudios epidemiológicos sobre la ETV en España. En este estudio, se describe la serie de casos de ETV diagnosticados en el único hospital de un área rural del sur de Extremadura (España) durante un periodo de 2 años.

Trabajo aceptado: 1 de junio de 2005 


\section{MATERIAL Y MÉTODOS}

Se realizó un estudio observacional prospectivo de todos los pacientes diagnosticados de ETV (TEP y/o TVP) mediante métodos objetivos entre noviembre de 2002 y noviembre de 2004 en el hospital de Zafra (Badajoz, Extremadura, España). El hospital de Zafra es un hospital de primer nivel que atiende una población fundamentalmente rural de 59.660 habitantes (30.449 hombres y 29.211 mujeres) según datos del censo del año 2003, y es el único centro hospitalario en su área de influencia.

El diagnóstico de TVP se estableció mediante ecografíadoppler venosa o tomografía computarizada (TC). El diagnóstico de TEP se estableció mediante gammagrafía pulmonar de ventilación/perfusión de alta probabilidad, TC o la asociación de un cuadro clínico compatible con TEP y la demostración de trombosis venosa profunda mediante ecografía-doppler venosa o TC. La investigación diagnóstica realizada dependió del criterio del médico responsable de cada paciente. En todos los pacientes se programó un seguimiento de al menos 3 meses con tratamiento anticoagulante.

Se consideró ETV “idiopática” si no existió ninguno de los factores de riesgo clásicos (cirugía mayor en los 2 últimos meses, inmovilización y/o cáncer activo). Se definió comorbilidad como la existencia de patología crónica grave pulmonar o cardíaca, demencia avanzada, cáncer activo u otras enfermedades graves. Se consideró hemorragia grave si causó un descenso de la hemoglobina superior a $2 \mathrm{~g} / \mathrm{dl}$, precisó de la transfusión de 2 o más concentrados de hematíes, o la localización fue retroperitoneal o en sistema nervioso central.

El análisis estadístico se realizó utilizando la prueba chicuadrado y el test exacto de Fisher, cuando alguno de los valores esperados fue menor de 5, para la comparación de proporciones. Se consideró estadísticamente significativo un valor de $\mathrm{p}<0,05$.

\section{RESULTADOS}

En el periodo estudiado se diagnosticaron de ETV un total de 76 pacientes: $35(46,1 \%)$ con TEP y $41(53,9 \%)$ con TVP aislada. El diagnóstico de TEP de estableció en 19 pacientes mediante gammagrafía pulmonar de ventilación/perfusión de alta probabilidad, en 15 pacientes mediante la asociación de un cuadro clínico compatible con TEP y la demostración de trombosis venosa profunda mediante ecografía-doppler venosa o TC, y en 1 paciente mediante TC. El diagnóstico de TVP aislada se estableció en 40 pacientes mediante ecografía-doppler venosa, y en 1 paciente mediante TC. Todos los pacientes excepto uno fueron de procedencia ambulatoria. En todos los casos la TVP se localizó en miembros inferiores. Entre los pacientes con TVP aislada, 29 (70,7\%) tuvieron una TVP proximal (localizada por encima del hueco poplíteo) y 12 $(29,3 \%)$ una TVP distal.

La incidencia anual de ETV fue 0,63 por 1.000 habitantes. La incidencia anual de ETV fue 0,74 por 1.000 habitantes en mujeres y 0,54 por 1.000 habitantes en hombres. La incidencia anual de TVP aislada fue 0,34 por 1.000 habitantes y de TEP 0,29 por 1.000 habitantes. El número de casos diagnosticados en los meses de diciembre-enero-febrero fue 19, en los meses de marzo-abril-mayo 16, en los meses de junio-julioagosto 22, y en los meses de septiembre-octubre-noviembre
19. La edad media de los pacientes fue 72,4 años (rango 35-94 años), $51(67,1 \%)$ tuvieron más de 70 años, y 43 (56,6\%) fueron mujeres. Treinta y siete pacientes $(48,7 \%)$ presentaron ETV “idiopática”. El resto de características epidemiológicas de la serie se describen en la tabla I.

\section{TABLA I}

\section{CARACTERÍSTICAS EPIDEMIOLÓGICAS DE LA SERIE}

\begin{tabular}{lr}
\hline & $n=76$ \\
\hline Inmovilización & $27(35,5 \%)$ \\
Cirugía general* $^{*}$ & $1(1,3 \%)$ \\
Cirugía traumatológica* $^{*}$ & $1(1,3 \%)$ \\
ETV previa & $5(6,6 \%)$ \\
Insuficiencia renal crónica** & $9(11,8 \%)$ \\
Comorbilidad & $32(42,1 \%)$ \\
Cáncer activo & $13(17,1 \%)$
\end{tabular}

ETV: enfermedad tromboembólica venosa. ${ }^{*}$ En los 2 últimos meses. * ${ }^{*}$ Creatinina plasmática $>1,5 \mathrm{mg} / \mathrm{dl}$.

Todos los pacientes, excepto 2 tratados con bemiparina y nadroparina, recibieron en la fase aguda enoxaparina subcutánea, habitualmente a dosis de $1 \mathrm{mg} / \mathrm{kg}$ de peso cada 12 horas o $1,5 \mathrm{mg} / \mathrm{kg}$ de peso cada 24 horas. Ningún paciente fue tratado con trombolíticos o un filtro en la vena cava inferior. El tiempo medio de seguimiento con tratamiento anticoagulante fue de 5,5 meses (rango 0-16 meses). Como tratamiento a largo plazo, 61 pacientes recibieron acenocumarol, 8 pacientes recibieron enoxaparina, y 2 recibieron inicialmente acenocumarol y posteriormente enoxaparina. Trece pacientes $(17,1 \%)$ fallecieron durante el seguimiento: 5 en la fase aguda y 8 tras un seguimiento medio de 2,4 meses (rango 1-5 meses). No se realizó autopsia en ninguno de los fallecidos. En la tabla II se comparan las características de los pacientes fallecidos y no fallecidos. Tres pacientes $(3,9 \%)$ presentaron sospecha clínica de recidiva tromboembólica, y todos ellos fallecieron. Cuatro pacientes $(5,3 \%)$ presentaron hemorragia grave no fatal (3 de localización gastrointestinal y 1 retroperitoneal), estando 3 en tratamiento con acenocumarol y 1 en tratamiento con enoxaparina. Nueve pacientes con TVP aislada no precisaron ingreso hospitalario, y no hubo ningún evento en el seguimiento.

\section{DISCUSIÓN}

La incidencia anual de ETV en nuestro área es inferior a la descrita en la mayoría de estudios de otros países occidentales, que se ha estimado en $0,71-1,83$ casos por 1.000 habitantes (2-5). Es posible que existan diferencias geográficas en la incidencia de ETV. No obstante, debe tenerse en cuenta que la actividad de cirugía traumatológica, un factor de riesgo importante para ETV, fue escasa en nuestro hospital en el periodo estudiado (sólo un paciente de nuestra serie tuvo el antecedente de cirugía traumatológica reciente), y es posible que pacientes de nuestra área hayan sido diagnosticados de ETV en hospitales de áreas limítrofes. Además, de forma general hay que tener en cuenta que el TEP es infradiagnosti- 

1. NOMBRE DE LA ESPECIALIDAD: CASENGLICOL. Polvo para Cada $100 \mathrm{~g}$ contiene: $P$ anhidro $(7,94 \mathrm{~g})$, bicarbonato sódico $(2,38 \mathrm{~g})$, cloruro sódico $(2,08 \mathrm{~g})$ ELECTROLITOS EN MILIÉOUIVALENTES (mEq/l): $\mathrm{Na}+(125,82 \mathrm{mEq})$, SO4 2- (78,87 mEq), HCO3 - (20,00 mEq), Cl - (35,00 mEq), H2PO4 - (4,08 $\mathrm{mEq}), \mathrm{K}+(10,30 \mathrm{mEq}), \mathrm{S} 205=(0,37 \mathrm{mEq})$. OSMOLARIDAD: 234, 97 $\mathrm{mOs} / \mathrm{l}$. Excipientes: ver punto 6.1. 3. FORMA para solución oral. 4. DATOS CLINICOS: 4.1. Indicaciones Terapéuticas: Evacuante intestinal para preparación previa a la colonoscopía, cirugía, radiología y otros exámenes colorectales genitourinarios. Tratamiento del estre organica que previamente ha sido tratado, sin resultados del ejercicio físico diario. 4.2. Posología y forma de administración: Administración oral. Para la evacuación intestinal con fines diagnósticos o cirugía: Adultos: Sobres de 70,5 g: La dosis completa es de 4 sobres de $70,5 \mathrm{~g}$. Disolver el contenido de un sobre en 1 litr g. Disolver el contenido La dosis completa . Disolver el conto cada 10-15 minutos hasta que la totalidad del volumen haya sido ingerido o la deposición sea clara. No se debe tomar ningún alimento durante las 3 ó 4 horas anteriores a la administración de la solución en ningún caso se ingerirá alimento sólido las 2 horas anteriores a la utilización de la misma. En pacientes incapaces de beber la solución se podrá utilizar sonda nasogástrica a razón de 20-30 ml por minuto. de haber iniciado el tratamiento. Niños: No hay experiencia clínica. Para el tratamiento del estreñimiento crónico de Adultos: A razónto del estreñimiento crónico de causa no orgánica: nerion resios cán dosificación in dositicacion en pacientes con insuficiencia hepática. Ancianos. La pauta posologica sería la misma que para adultos. Como para todos Polvo para solución oral. No utilizar Casenglicol Polvo para solución oral durante más de seis dís sin consultar con su médico. 4.3. Contraindicaciones: La administración de Casenglicol Polvo para solución oral está contraindicada en pacientes con: obstrucción y perforación gastrointestinal, retención gástrica, enfermedad intestinal inflamatoria cronica, megacolon, ileo, úlcera gástrica intestinal. Hipersensibilidad a los principios activos 0 a cualquiera de los componentes del producto. 4.4. Advertencias y precauciones especiales de empleo: Durante la administración de Casenglico Polvo para solución oral, especialmente mediante sonda nasogástrica, debe vigilarse para evitar la regurgitación o aspiración

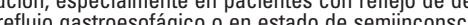
inconsciencia Sistoesofágico en abdominal, la administración se realizará más lentamente 0 , incluso, se interrumpirá hasta la reversion de los síntomas. Si se sospecha la existencia de obstrucción o perforación gastrointestinal, se deben llevar a cabo las exploraciones diagnósticas necesarias antes de la administración de Casenglicol Polvo para solución oral. Casenglicol Polvo para solución oral debe emplearse con precaución en pacientes con colitis severa o proctitis. Este medicamento contiene como excipiente metabisulfito sodico, por lo que puede causar reacciones de tipo alérgico, incluyendo reacciones anafilácticas o broncoespasmo en pacientes susceptibles, especialmente en aquellos con historial asmatico alérgico. 4.5. Interacciones con otros medicamentos y otras formas de interacción: La medicación administrada durante la utilización de Casenglicol Polvo para solución oral podría eluirse por el tracto gastrointestinal y no absorberse. 4.6. Embarazo y lactancia: No exist durante el embarazo y lactancia. No debe ser empleada durante el 4.7. Efectos sobre la capacidad de conducir y utilizar maquinaria: $\mathrm{No}$ han sido descritos. 4.8. Reacciones adversas: Náuseas, sensación de plenitud abdominal y retortijones son las reacciones más comunes. abdominales e irritación anal. Todas estas reacciones adversas son transitorias y ceden rápidamente. Han sido descritos algunos casos alérgicas. 4.9. Sobredosificación: No procede, ya que los 5. PROPIEDADES FARMACOLOGICAS. 5.1. Propiedades farmacodinámices: Grupo farmoterántic: A06AD: laxantes osmóticos. La solución preparada induce a una rápida evacuación intericida osmótica del PEG 4000 y la concentración de electrolitos resultan en una no absorción o excreción netas de agua o iones. Como consecuencia, pueden ser administrados grandes volúmenes de solución sin cambios significativos en el balance hidroelectrolítico de organismo. 5.2. Propiedades farmacocinéticas: Casenglicol Polvo para solución oral es una solución isotónica compuesta por el agente osmótico polietilenglicol 4.000 y electrolitos. Al ser isotónica, la 5.3. Datos preclínicos sobre seguridad: Las soluciones a base de polietilenglicol y de electrolitos han sido utilizadas ampliamente y está demostrada su seguridad de uso. 6. DATOS FARMACÉUTICOS: 6.1. Relación de excipientes: Metabisulfito sodico, aroma de naranja, acesulfamo, sacarina sódica. 6.2. Incompatibilidades: No se han
descrito. 6.3. Período de validez: 3 años. 6.4. Precauciones especiales de conservación: Mantener por debajo de los $30^{\circ} \mathrm{C}$. Un 2 y $8^{\circ} \mathrm{C}$ hasta 48 horas. 6.5 . Naturaleza y contenido del recipiente: La especialidad se presenta en sobres monodosis de poliester/aluminio / polietileno con un contenido de $70,5 \mathrm{~g}$ o de $17,6 \mathrm{~g}$ de polvo por sobre según la presentacion. Presentaciones: 4 sobres de 70,5 g, 100 sobres de 70,5 $\mathrm{g}, 16$ sobres de $17,6 \mathrm{~g}$ y 500 sobres de 17,6 g. 6.6. Instrucciones de uso manipulación: Cada sobre de 70,5g se debe disolver en 1 litro de agua. La dosis total es de 4 sobres. Cada sobre de $17,6 \mathrm{~g}$ se debe preparada debe consumirse en el término de 4 horas. 7. TITULAR DE LA AUTORIZACION: LABORATORIOS CASEN FLEET. Autovía de Logroño, Km 13,300. 50180-UTEBO, ZARAGOZA (ESPANA).
8. NUMERO DE AUTORIZACION DE COMERCIALIZACIÓN: Registrado 政 4 sobres de 70,5 g: PVL 6,90€/ PVP $10,59 € /$ PVP (IVA) 11,01€. Envase Clínico de 100 sobres de 70,5 g: PVL PVL 6,90€/ PVP 10,59€/ PVP(IVA) $11,01 €$. Envase Clínico de 500 sobres de 17,6 g: PVL 179,43€ 9. FECHA DE FEBRERO 2003. 10. FECHA DE REVISION DEL TEXTO: NOVIEMBRE 2003. 11 CONDICIONES DE PRESCRIPCION: Con

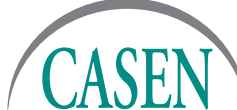

Frept

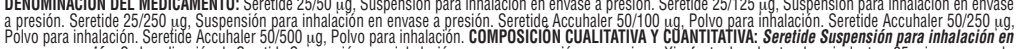
envase a presión. Cada aplicación de Seretide Suspensión para inhalación en envase a presión proporciona: Xinatoato de salmeterol equivalente a 25 microgramos de microgramos de propionato de fluticasonana liberados del aplicador (dosis liberada). Excipientes. Norftlurano (HFA 134a). Seretide Accuhalerer. Cada dosis de Seretide

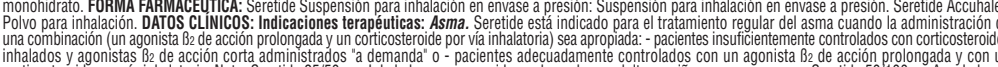
corticosteroide por vía inhalatoria. Nota: Seretide $25 / 50 \mu \mathrm{ug}$, Inhalador no se considera adecuado en adultos y ninos con asma grave. Seretide $50 / 100 \mathrm{ng}$ A Accuhaler $n$

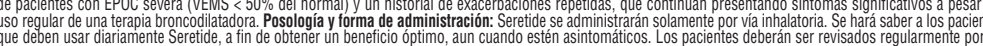
más baja con la que se mantenga un control eficaz de los sintomas. Cuando el control de los sintomas se mantenga con la dosis más baja de la combinación administrad
dos veces al día, entonces el siguiente paso podría consistir en probar el tratamiento exclusivamente con un corticosteroide por vía respiratoria. Como alternati aquellos pacientes que precisen de un agonistata $\beta_{2}$ de acción prolongada podrán recibir Seretide una vez al día si en opinión de su $\mathrm{m}$.

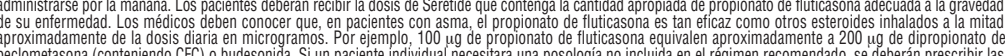

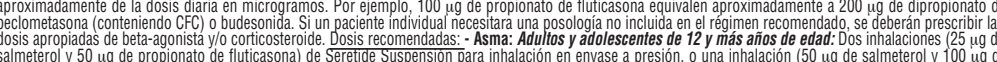

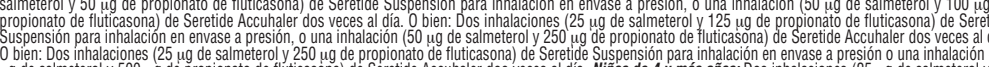

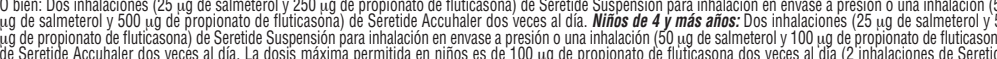
$25 / 50$, Suspensión para inhalación en envase a presión dos veces al días o una inhalación de Seretide Accuhaler 50/100 $\mu \mathrm{g}$, Polvo para inhalación dos vecesel al diar.

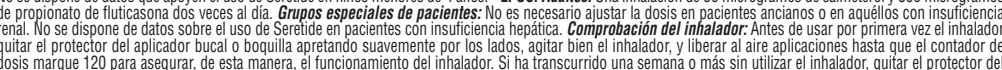

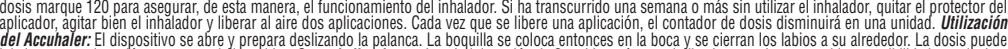

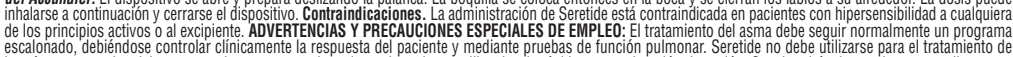

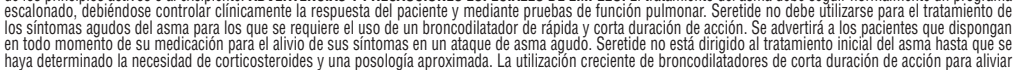

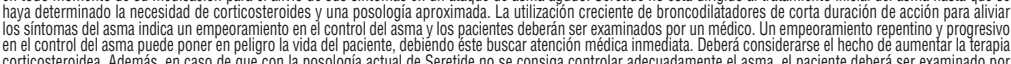
un médico. Deberá considerarse la posbililidad de incluir terapias corticosteroideas adicionales. El tratamiento con Seretide no, debe suspenderse bruscamente. Conn

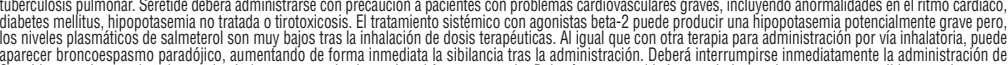

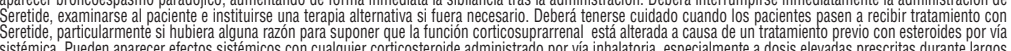

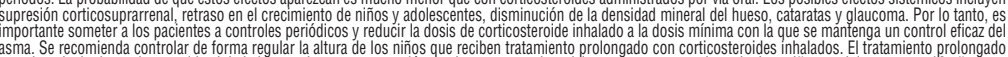
con altas dosis de corticosteroides inhalados puede causar suppresión corticicosuprarrenal y crisis corticosurrarrenal aguda. Los niños y adolescentes < 16 años que

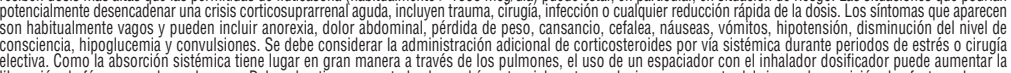

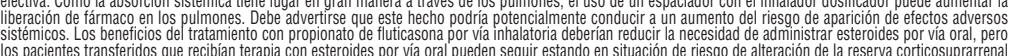

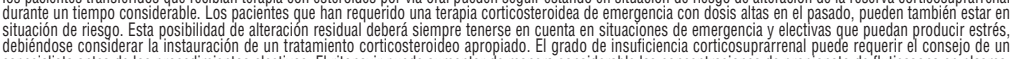

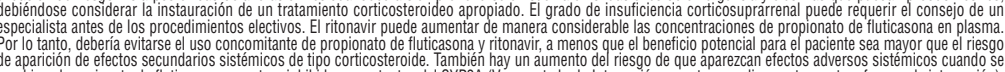

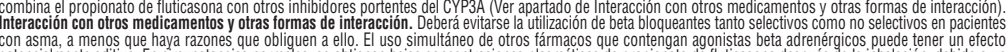

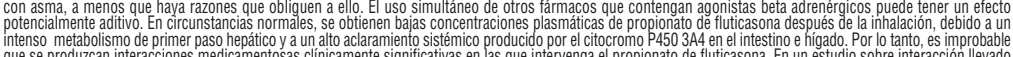

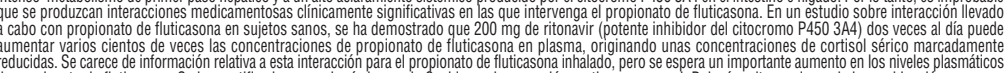
de propionato de fluticasona. Se han notificado casos de sindrome de Cushing y de supresión corticosuprarrenal. Debería evitarse el uso de la combinación, a menos

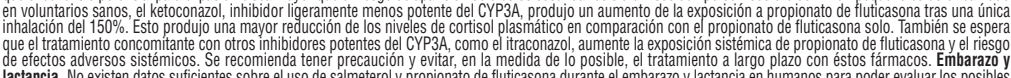
de efectos adversos sistémicos. Se recomienda tener precaución y evitar, en la medida de lo posible, el tratamiento a largo plazo con éstos fármacos. Embarazo y
lactancia. No existen datos suficientes sobre el uso de salmeterol y propionato de fluticasona durante el embarazo y lactancia en humanos para poder evaluar los posibles
efectos nocivos. En estudios realizados con animales,

\begin{tabular}{|c|c|c|}
\hline Clasificación de órganos & \begin{tabular}{|l|} 
Efecto adverso \\
\end{tabular} & \begin{tabular}{|l} 
Frecuencia \\
\end{tabular} \\
\hline Infecciones e infestaciones & Candidiasis en la boca y garganta & \begin{tabular}{|l} 
Frecuentes \\
\end{tabular} \\
\hline $\begin{array}{l}\text { Trastornos del sistema } \\
\text { inmunológico }\end{array}$ & \begin{tabular}{|l|} 
Reacciones de hipersensibilidad con las siguientes \\
manifestaciones: \\
Reacciones de hipersensibilididad cutánea \\
Angioedema (principalmente edema facial y orofaríngeo), sintomas \\
respiratorios (disnea y/o broncoespamo) y reacciones anafilácticas
\end{tabular} & $\begin{array}{l}\text { Poco frecuentes } \\
\text { Muy raros }\end{array}$ \\
\hline Trastornos endocrinos & $\begin{array}{l}\begin{array}{l}\text { Supresión corticosuprarrenal, retraso en el crecimiento de niños } \\
\text { y adolescentes, dismininución de la densidad mineral del hueso, } \\
\text { cadtaratas y glaucoma. }\end{array} \\
\end{array}$ & Muy raros \\
\hline Trastornos del sistema nervioso & $\begin{array}{l}\text { Cefalea } \\
\text { Temblor }\end{array}$ & $\begin{array}{l}\text { *Muy frecuente } \\
\text { Frecuente }\end{array}$ \\
\hline Trastornos cardiacos & \begin{tabular}{|l|} 
Palpitaciones \\
Taquicardia \\
Arritmias cardiacas (incluyendo fibrilación auricular, taquicardia \\
supraventricular y extrasistoles)
\end{tabular} & $\begin{array}{l}\text { Frecuentes } \\
\text { Poco frecuente } \\
\text { Muy raras }\end{array}$ \\
\hline $\begin{array}{l}\text { Trastornos respiratorios, torácicos } \\
\text { y mediastinicos }\end{array}$ & \begin{tabular}{|l|l|} 
Irritación de garganta \\
Ronquera/ disfonía \\
Broncoespasmo paradójico \\
\end{tabular} & $\begin{array}{l}\text { Frecuente } \\
\text { Frecuentes } \\
\text { Muy raro }\end{array}$ \\
\hline $\begin{array}{l}\text { Trastonos musculoesqueléticos y del } \\
\text { tejido conjuntivo }\end{array}$ & \begin{tabular}{|l|} 
Calambres musculares \\
Atralgia \\
Mialgia
\end{tabular} & $\begin{array}{l}\text { Frecuentes } \\
\text { Muy rara } \\
\text { Muy rara }\end{array}$ \\
\hline
\end{tabular}

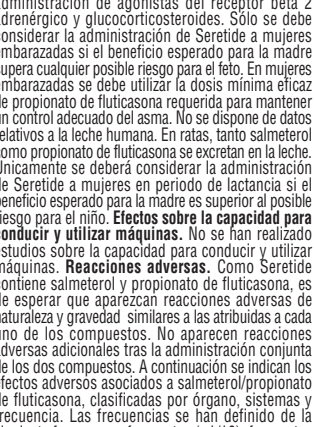

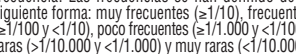
incluyendo notificaciones aisladas. Las reacciones muy frecuentes, frecuentes y poco frecuentes se han identificado generalmente a partir de los datos de ensayos
clinicos. No se ha tenido en cuenta la incidencia en el caso del placebo. Los acontecimientos muy raros proceden generalmente de datos notificados espontaneamente postcomercialización. Se han comunicado efectos secundarios farmacológicos de un tratamiento con un agonista beta-2. tales como temblor, palpitaciones y cefalea,
pero tienden a ser transitorios y disminuyen con una terapia regular. Debicio al componente propionato de fluticasona, algunos pacientes pueden padecer ronqueray

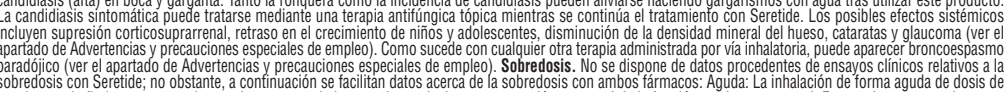

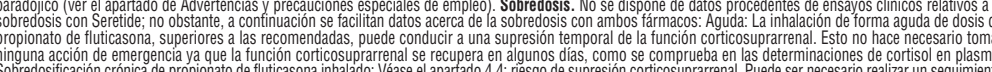

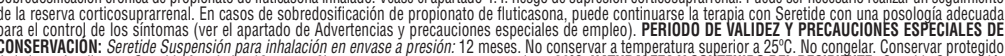
CONSERVACION: Seretide SUSpensión para inhalación en envase a presión: 12 meses. No conservar a temperatura superior a $25^{\circ}$ C. No congelar: Conservar protegido
de la luz solar directa. Seretide Accuhaler: 18 meses. No conservar a temperatura superior a $30^{\circ}$. NATURALEZA Y CONTENIDO DELL RECIPIENTE: Seretide Suspensión

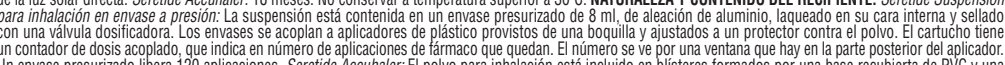

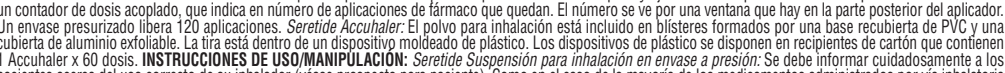

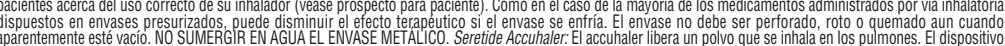

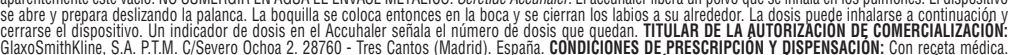

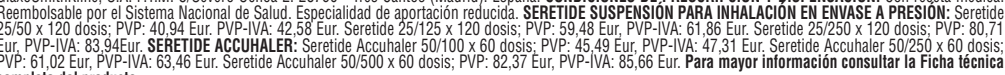


TABLA II

COMPARACIÓN DE LAS CARACTERÍSTICAS DE LOS PACIENTES FALLECIDOS Y NO FALLECIDOS

\begin{tabular}{lcccc}
\hline & $\begin{array}{c}\text { Fallecidos } \\
n=13\end{array}$ & $\begin{array}{c}\text { No fallecidos } \\
n=63\end{array}$ & $p$ & OR (IC 95\%) \\
\hline Edad > 70 años & $10(76,9 \%)$ & $41(65,1 \%)$ & NS & $1,8(0,4-7,2)$ \\
Sexo femenino & $9(69,2 \%)$ & $34(54 \%)$ & NS & $1,9(0,5-6,9)$ \\
TEP & $8(61,5 \%)$ & $27(42,9 \%)$ & NS & $2,1(0,6-7,3)$ \\
ETV no "idiopática" & $12(92,3 \%)$ & $27(42,9 \%)$ & 0,001 & $16(1,9-130,7)$ \\
Insuficiencia renal crónica* & $3(23,1 \%)$ & $6(9,5 \%)$ & NS & $2,8(0,6-13,3)$ \\
Comorbilidad & $11(84,6 \%)$ & $21(33,3 \%)$ & 0,0008 & $11(2,2-54,2)$ \\
Cáncer activo & $8(61,5 \%)$ & $5(7,9 \%)$ & $<0,0001$ & $18,6(4,4-78,6)$
\end{tabular}

TEP: tromboembolismo pulmonar; ETV: enfermedad tromboembólica venosa; *Creatinina plasmática > 1,5 mg/dl; OR: odds ratio; IC: intervalo de confianza. NS: no significación estadísti$\mathrm{ca}(\mathrm{p} \geq 0,05)$.

cado $(1,6)$, y que la frecuencia de realización de autopsias (que en nuestro hospital es muy baja) también influye en la incidencia estimada de TEP y por tanto de ETV. La mayoría de los estudios epidemiológicos de ETV demuestran una mayor proporción de pacientes con TVP aislada que con TEP (2-5), como ocurrió en nuestro estudio. Esta proporción está influida por lo exhaustivo de la investigación diagnóstica en la búsqueda de TEP en pacientes con TVP y por los criterios utilizados para establecer el diagnóstico de TEP. En nuestro estudio, no encontramos diferencias estacionales destacables en la incidencia de ETV. Por el contrario, en un estudio en Francia de más de 120.000 ingresos hospitalarios por ETV hubo un mayor número de diagnósticos en los meses de invierno (7).

La ETV afecta preferentemente a ancianos $(2-5,8)$, como ocurrió en nuestro estudio. La incidencia de ETV parece no ser significativamente distinta en hombres y mujeres en la mayoría de estudios (2), aunque en el nuestro sí fue considerablemente mayor en mujeres. La frecuencia de ETV "idiopática" se ha estimado en un 26-47\%, dependiendo de la definición utilizada (2). Casi la mitad de nuestros pacientes tuvieron una ETV "idiopática” (no asociada a factores de riesgo clásicos: cirugía mayor en los 2 últimos meses, inmovilización y/o cáncer activo). Por tanto, la ausencia de estos factores de riesgo en pacientes con ETV es frecuente en nuestro medio. Aproximadamente un tercio de nuestros pacientes tuvieron el antecedente de inmovilización, y sólo una minoría el antecedente de ETV previa. Más de un tercio de nuestros pacientes tuvieron comorbilidad asociada, y concretamente casi una sexta parte presentó cáncer activo. Estos datos sugieren la importancia de considerar el diagnóstico de ETV en estos pacientes. Por ejemplo, 142 pacientes $(22,7 \%)$ tuvieron cáncer activo en una serie de Estados Unidos (EE.UU.) de 625 pacientes con ETV (9).

La mortalidad tras un seguimiento medio de más de 5 meses con tratamiento anticoagulante fue considerable en nuestra serie, y puede considerarse algo distinta a la descrita en otros estudios. En un estudio en EE.UU., la mortalidad al año en pacientes con ETV fue del 19\% (5). En el Registro Informatizado de Enfermedad Tromboembólica Venosa en España (RIETE), la mortalidad tras un seguimiento medio de 5 meses fue del 12,5\% (10). Como cabría esperar, la existencia de TEP fue más frecuente en nuestros pacientes fallecidos que en los no fallecidos, aunque sin alcanzar la significación estadística. La ETV no "idiopática" fue significativamente más frecuente en nuestros pacientes fallecidos, lo que sugiere que la presencia de factores de riesgo clásicos (cirugía mayor en los 2 últimos meses, inmovilización y/o cáncer activo) se asocia a un peor pronóstico. En este sentido, igualmente la presencia de comorbilidad, incluido el cáncer activo, fue significativamente más frecuente en nuestros pacientes fallecidos. En un estudio de pacientes mayores de 45 años con ETV en EE.UU., el único factor asociado a un aumento de la mortalidad a los 28 días fue la presencia de cáncer (11). Estos resultados pueden indicar que la mortalidad en la ETV está relacionada en gran parte con la situación basal previa del paciente.

Una limitación de nuestro estudio es que no se realizó autopsia en ninguno de los pacientes fallecidos, por lo que no tenemos certeza de las causas de las muertes. No obstante, en casi el $4 \%$ se estableció la existencia de probable recidiva tromboembólica y todos los pacientes fallecieron. En una cohorte de EE.UU. de más de 70.000 pacientes con ETV, la frecuencia de recidiva tromboembólica en los 6 primeros meses fue de aproximadamente un $6 \%$, algo superior a la de nuestro estudio (12). La recidiva tromboembólica durante el tratamiento anticoagulante parece no ser excepcional en nuestro medio, y cuando ocurre puede tener un pronóstico fatal. La frecuencia de hemorragia grave no fatal durante el seguimiento con tratamiento anticoagulante no fue despreciable en nuestros pacientes, aproximadamente del 5\%. Esta frecuencia es considerablemente superior a la descrita en un meta-análisis de ensayos clínicos en el que la frecuencia de hemorragia grave fatal o no fatal fue aproximadamente del 2,5\%, aunque durante un seguimiento más corto, los 3 primeros meses de tratamiento anticoagulante (13). En el RIETE, la frecuencia de hemorragia grave tras un seguimiento medio de 5 meses con tratamiento anticoagulante fue de un 4,3\% (10), muy similar a la de nuestro estudio. Los resultados de nuestro estudio apoyan la observación de otros estudios de una mayor frecuencia de complicaciones hemorrágicas asociadas a la anticoagulación en pacientes con ETV en la práctica clínica habitual que en los ensayos clínicos (14).

Por último, indicar que el tratamiento ambulatorio de la TVP aislada puede ser una opción en casos seleccionados, como se comprobó en 9 de nuestros pacientes, y como se ha demostrado en estudios previos (15). 


\section{Bibliografía}

1. Kearon C. Natural history of venous thromboembolism. Circulation 2003; 107 (Supl. 1): 22-30.

2. White RH. The epidemiology of venous thromboembolism. Circulation 2003; 107 (Supl. 1): 4-8.

3. Silverstein MD, Heit JA, Mohr DN, Petterson TM, O'Fallon WM, Melton LJ III. Trends in the incidence of deep vein thrombosis and pulmonary embolism: a 25-year population-based study. Arch Intern Med 1998; 158: 585-93.

4. Oger E. Incidence of venous thromboembolism: a community-based study in Western France. EPI-GETBP Study Group. Groupe d'Etude de la Thrombose de Bretagne Occidentale. Thromb Haemost 2000; 83: 657-60.

5. Anderson FA Jr, Wheeler HB, Goldberg RJ, Hosmer DW, Patwardhan NA, Jovanovic B, et al. A population-based perspective of the hospital incidence and case-fatality rates of deep vein thrombosis and pulmonary embolism. The Worcester DVT Study. Arch Intern Med 1991; 151: 933-8.

6. Morgenthaler TI, Ryu JH. Clinical characteristics of fatal pulmonary embolism in a referral hospital. Mayo Clin Proc 1995; 70: 417-24.

7. Boulay F, Berthier F, Schoukroun G, Raybaut C, Gendreike Y, Blaive B. Seasonal variations in hospital admission for deep vein thrombosis and pulmonary embolism: analysis of discharge data. BMJ 2001; 323 : 601-2.

8. Calvo Romero JM, Pérez Miranda M, Bureo Dacal P. Tromboembolismo pulmonar en el anciano. An Med Interna (Madrid) 2003; 20: 21-4.
9. Heit JA, Silverstein MD, Mohr DN, Petterson TM, O'Fallon WM, Melton LJ III. Risk factors for deep vein thrombosis and pulmonary embolism: a population-based case-control study. Arch Intern Med 2000; 160: 809-15.

10. Arcelus JI, Caprini JA, Monreal M, Suárez C, González-Fajardo J. The management and outcome of acute venous thromboembolism: a prospective registry including 4011 patients. J Vasc Surg 2003; 38: 916-22.

11. Cushman M, Tsai AW, White RH, Heckbert SR, Rosamond WD, Enright $\mathrm{P}$, et al. Deep vein thrombosis and pulmonary embolism in two cohorts: the longitudinal investigation of thromboembolism etiology. Am J Med 2004; 117: 19-25.

12. Murin S, Romano PS, White RH. Comparison of outcomes after hospitalization for deep venous thrombosis or pulmonary embolism. Thromb Haemost 2002; 88: 407-14.

13. Linkins LA, Choi PT, Douketis JD. Clinical impact of bleeding in patients taking oral anticoagulant therapy for venous thromboembolism: a meta-analysis. Ann Intern Med 2003; 139: 893-900.

14. Willey VJ, Bullano MF, Hauch O, Reynolds M, Wygant G, Hoffman L, et al. Management patterns and outcomes of patients with venous thromboembolism in the usual community practice setting. Clin Ther 2004; 26: 1149-59.

15. Segal JB, Bolger DT, Jenckes MW, Krishnan JA, Streiff MB, Eng J, et al. Outpatient therapy with low molecular weight heparin for the treatment of venous thromboembolism: a review of efficacy, safety, and costs. Am J Med 2003; 115: 298-308. 